\title{
Vacuolar segregation to the bud of Saccharomyces cerevisiae: an analysis of morphology and timing in the cell cycle
}

\author{
Daniel S. Gomes de Mesquita, ${ }^{*}$ Rogier ten Hoopen and ConRad L. Woldringh \\ Department of Molecular Cell Biology, Section of Molecular Cytology, University of Amsterdam, Plantage Muidergracht \\ 14, 1018 TV Amsterdam, The Netherlands
}

(Received 7 May 1991; revised 3 July 1991; accepted 8 July 1991)

\begin{abstract}
Vacuoles of Saccharomyces cerevisiae were visualized by phase-contrast microscopy. Visualization was enhanced by adding polyvinylpyrrolidone. Vacuolar segregation during the cell cycle was analysed in $\mathbf{4 2}$ individual cells of strain X2180 by time-lapse photomicrography. Within $15 \mathrm{~min}$ of bud emergence, more than $80 \%$ of the cells contained a vacuolar segregation structure in the form of either a tubule or an alignment of vesicles. The structure emerged from one point of the mother vacuole, then elongated and moved into the bud in a few minutes. The vacuolar segregation structure disappeared, usually within $20 \mathrm{~min}$, before nuclear migration, leaving a separate vacuole in the bud. To test the generality of this observation several strains were grown in the presence of the vacuolar vital dye fluorescein isothiocyanate. The bud size was used to measure progress in the cell cycle. All strains formed vacuolar segregation structures in cells with small buds, although with variations in duration and timing in the cell cycle. In the presence of nocodazole vacuolar segregation occurred normally, thus, microtubules seem not to be essential in this process.
\end{abstract}

\section{Introduction}

Vacuoles are involved in diverse processes, such as protein degradation, metabolite storage, ion homeostasis and osmoregulation (reviewed by Klionsky et al., 1990). Recently, vacuoles of Saccharomyces cerevisiae have been the subject of studies on organelle inheritance (Weisman et al., 1987, 1990; Raymond et al., 1990). In living cells vacuoles can be seen by phase-contrast microscopy or by fluorescence microscopy, using one of the vacuolar staining procedures (Pringle et al., 1989). This facilitates monitoring the way in which vacuoles reach their new location in the bud.

Two previously published results indicated that vacuoles in the bud might be derived from part of the mother vacuole that segregates early in the cell cycle. First, Weisman \& Wickner (1988) made use of a red fluorophore that accumulated in the vacuole in ade2 mutants during stationary phase. They showed that in zygotes, with only one parental vacuole stained, the fluorophore remained restricted to that vacuole. In mitotic cells the fluorophore is probably also retained

Abbreviations: DAPI,4',6-diamidino-2-phenylindole; FITC, fluorescein isothiocyanate; PVP, polyvinylpyrrolidone. within the original vacuole. Stained cells that were reincubated in fresh medium with adenine stopped synthesizing the fluorophore, but new bud vacuoles still received the fluorophore, although at a lower concentration than the mother vacuole (Weisman et al., 1987). This indicated that part of the bud vacuole is derived from the mother vacuole. Second, Raymond et al. (1990) showed that the vacuole was lying in the neck in cells with buds of intermediate size. In small buds no detectable vacuole was present and large buds had a separate vacuole. The vacuolar structure in the neck is probably the structure by which mother vacuolar content is brought to the bud, and Raymond et al. (1990) termed it the vacuole segregation structure.

Several recently characterized mutations have some effect on vacuolar segregation. Some of the mutants also have an altered vacuolar morphology: one large round vacuole instead of 3-30 smaller vesicles (Raymond et al., 1990). None of the known vacuole mutations clearly blocked vacuolar segregation to the bud. Vacuolar segregation is delayed in vps33 and vps34 mutants (Banta et al., 1990; Herman \& Emr, 1990). This might be an indirect effect because these mutants also have a distorted vacuolar biogenesis. As a result, other proteins needed for segregation might be present at too low 
concentrations in the vacuole. In vps 3 and vacl mutants very little of the mother vacuole segregated to the bud (Raymond et al., 1990; Weisman et al., 1990). Some bud vacuoles can hardly be seen by fluorescence microscopy and others are probably present but can not be seen. Therefore, it is difficult to measure the timing of vacuolar segregation in these mutants, but at least some small buds with a segregation structure and with separate vacuoles were seen at much the same bud size as in wildtype cells (Raymond et al., 1990).

In this paper we give a quantified description of the timing of vacuolar segregation and the accompanying changes in vacuolar morphology in wild-type cells of $S$. cerevisiae. This might be important for interpreting results from mutants with a defect in vacuolar segregation. We show that the vacuolar segregation structure is an elongation of the mother vacuole directed to the bud.

\section{Methods}

Yeast strains and chemicals. The Saccharomyces cerevisiae strains used were as follows: X2180 a/ $\alpha$ CUP1/CUPI $\mathrm{gal} / \mathrm{gal} 2 \mathrm{mal} / \mathrm{mal} \mathrm{mel} / \mathrm{mel}$ SUC2/SUC2; S288C a CUP1 gal2 mal mel SUC2; DBY747 a canl gal his3- $\Delta$ l leu2-3 leu2-112 trpl-289 ura3-52 (all obtained from the Yeast Genetic Stock Center); D273-10B $\alpha$ (from A. Tzagoloff, Department of Biological Sciences, Colombia University, New York, NY, USA) and DLl $\alpha$ his3 leu 2 ura3 (described by Van Loon et al., 1983). Fluorescein isothiocyanate isomer I (FITC) was obtained from Serva; 4, 6diamidino-2-phenylindole (DAPI) and methyl-(5-[2-thienylcarbonyl]1H-benzimidazol-2-yl) carbamate (nocodazole) from Sigma and polyvinylpyrrolidone K90 (PVP) from Fluka.

Growth conditions, staining and cell number counting. All strains were grown on YPD [1\%(w/v) yeast extract, $2 \%(w / v)$ peptone, $2 \%(w / v)$ glucose] adjusted to $\mathrm{pH} 5$ with $\mathrm{HCl}$. Prior to each experiment cells were kept in exponential phase $\left(\mathrm{OD}_{450}<1\right)$ for more than 20 generations by periodic dilution of batch cultures. FITC staining was performed in the growth medium for $15 \mathrm{~min}$ or more at a final concentration of $10 \mu \mathrm{g}$ $\mathrm{ml}^{-1}$ (diluted from $1 \mathrm{mg} \mathrm{ml}^{-1}$ stock in $30 \mathrm{~mm}$-Tris/ $\mathrm{HCl}, \mathrm{pH} 8$ ). Cells were concentrated by $15 \mathrm{~s}$ centrifugation at $13000 \mathrm{~g}$ and directly prepared for microscopy. DAPI staining was performed on cells fixed in the growth medium by $0.25 \%$ formaldehyde. They were washed in

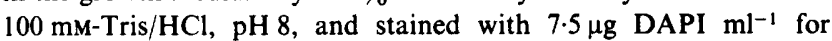
$30 \mathrm{~min}$. Cell numbers were measured with a Coulter counter equipped with a $70 \mu \mathrm{m}$ orifice.

Microscopy and photography. Fluorescence microscopes of Zeiss (Photomicroscope II) and Leitz (Dialux) were used with $100 \times$ phasecontrast neofluar oil-immersion lenses (numeric aperture $=1 \cdot 30$ ). All photographs were made on Kodak TMY400 film, processed as 1600 ASA. In most fluorescence photomicrographs the cell contours were visualized in the same picture by using a dimmed background of phasecontrast illumination.

Confocal scanning laser microscopy. FITC-stained vacuoles of X2180 were visualized in the confocal scanning laser microscope developed in our laboratory (Brakenhoff et al., 1989), using the $482 \mathrm{~nm}$ excitory band from a krypton-ion laser. The images were filtered with a 3DGauss filter and the background was removed by thresholding and contrast stretching (Gonzalez \& Wintz, 1987) on an HP Apollo DN 4000 workstation. The $3 \mathrm{D}$-image of the FITC-stained compartment was visualized with the simulated fluorescence procedure developed by van der Voort et al. (1988), which produces a picture with artificial shadows and background. The cell contour was negatively stained by FITC in the medium around the cells.

Time-lapse experiments. Cells of strain X2180 were concentrated by centrifugation $(15 \mathrm{~s}, 13000 \mathrm{~g})$ and spread on an object slide coated with

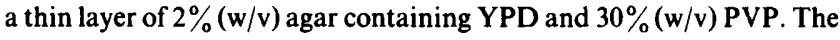
PVP was added to give better visualization of vacuoles by phasecontrast microscopy (Robinow, 1975; Lord \& Wheals, 1981). A coverslip on the cells was sealed at two joining sides with nail polish. The third side was left open for aeration and at the fourth side a wet filter paper was attached for hydration. Cells, lying near the corner of the aeration and hydration sites, were followed during their cell cycle under the microscope in a $30^{\circ} \mathrm{C}$ room. Photographs were taken about every $4 \mathrm{~min}$ and vacuolar segregation was scored from the pictures.

Cell synchronization. Cells were grown to $\mathrm{OD}_{450}=1\left(\sim 6 \times 10^{6}\right.$ cells $\left.\mathrm{ml}^{-1}\right)$. One litre was centrifuged for $5 \mathrm{~min}$ at $3000 \mathrm{~g}$ and resuspended in $5 \mathrm{ml}$ growth medium. The cells were injected into a Beckman JE-6 centrifugal elutriation rotor at 2700 r.p.m. The flow of medium $\left(30^{\circ} \mathrm{C}\right)$ was kept at $10 \mathrm{ml} \mathrm{min}^{-1}$ for $30 \mathrm{~min}$. Cell fractions were obtained by gradually increasing the flow rate to $15 \mathrm{ml} \mathrm{min}^{-1}$. An $80-100 \mathrm{ml}$ fraction with mostly unbudded cells was used.

Nocodazole treatment. Nocodazole was dissolved in dimethyl sulphoxide $\left(5 \mathrm{mg} \mathrm{ml}^{-1}\right)$ and added to the culture at a final concentration of $12.5 \mu \mathrm{g} \mathrm{ml}^{-1}$, as described by Guthrie \& Wickner (1988).

\section{Results}

\section{FITC staining of vacuoles}

Vacuoles were stained with FITC in the growth medium. FITC is suspected to be a physiologically perturbing dye (Preston et al., 1987; Pringle et al., 1989). However, its suspected reactivity occurs in vitro only at unphysiologically high $\mathrm{pH}(>8)$. Cells grown in the presence of FITC $\left(10 \mu \mathrm{g} \mathrm{ml}^{-1}\right)$ for more than $5 \mathrm{~h}$ did not show any change in growth rate, as measured by $\mathrm{OD}_{650}$ and cell number increase, and they continued to grow in FITC-containing medium for at least several days. The cell morphology was unaffected by FITC as seen by light microscopy. As illustrated in Fig. 1, the vacuolar shape looked similar by phase-contrast microscopy and by fluorescence microscopy, except that the vacuoles appeared more slender in phase-contrast microscopy.

Only irradiation of FITC-stained cells with intense blue light affected the vacuolar morphology, causing fusion of vacuolar vesicles. We do not know whether intense blue light has the same effect in unstained cells, but it has been reported that anaerobic conditions can cause fusion of vacuoles (Pringle et al., 1989). In media of $\mathrm{pH} \geqslant 7$, or in cells in stationary phase, FITC did not effectively stain the vacuoles. Likewise, in tetrads, FITC did not stain the vacuoles, but it stained the ascospore wall. Since FITC had no visible effect on cells, but stained vacuoles more easily and brightly than most other stains (e.g. Lucifer yellow carbohydrazide, and 5- 
(a)
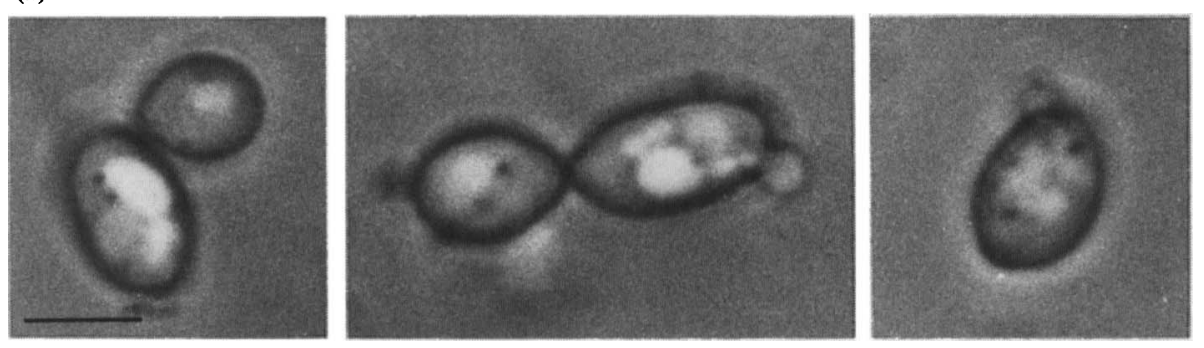

(b)
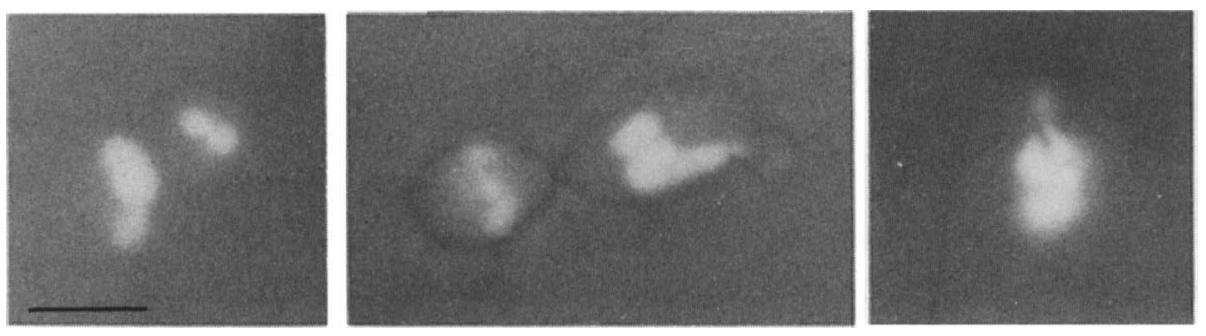

Fig. 1. Comparison of vacuoles of FITCstained X2180 cells, growing on YPD-agar with $30 \%$ PVP, as seen by phase-contrast microscopy (a) and fluorescence microscopy (b). Small differences in vacuolar structure might be due to vacuolar movements in the interval between the two pictures $(\sim 0.5 \mathrm{~min})$. Bars, $5 \mu \mathrm{m}$.

and 6-carboxyfluorescein diacetate; data not shown), we used it in all our experiments except the time-lapse experiments, where phase-contrast microscopy was used.

\section{Vacuolar segregation in individual cells}

For time-lapse photography $\mathrm{X} 2180$ cells were grown on YPD-agar under the microscope and followed during their cell cycle. FITC staining could not be used because it bleached after two to four exposures, whereas about 30 pictures per cell cycle were taken. To enhance the phase contrast of vacuoles PVP was added to the agar, thereby increasing the refractive index of the agar. With this method vacuoles could be seen as areas of low refractive index (see Fig. $1 a$ ) in $80 \%$ of the cells. The vacuoles in the other $20 \%$ might have had a higher refractive index, or alternatively, these vacuoles consisted of vesicles too small to be seen.

In 42 cells vacuolar morphology was followed from bud emergence to cell division (Table 1). In the average cell no vacuole was seen in the bud until approximately $5 \mathrm{~min}$ after bud emergence. In some cells a tubular extension was seen to be pulled out of the mother vacuole and extended through the neck. Such a tubular extension, shown in Fig. $2(t=23-54 \mathrm{~min})$, has been designated the vacuole segregation structure (Raymond et al., 1990). In photomicrographs, only nine cells showed the stadium with the vacuole pointing towards the bud, but not yet lying in the neck (Fig. $2, t=10 \mathrm{~min}$ ), but it was seen more often in the microscope. In most cells, the formation of the segregation structure occurred within the period of a few minutes between two successive photomicrographs. The vacuolar segregation structure
Table 1. Timing of the vacuolar segregation in X2180 cells growing on YPD-agar with $30 \%$ PVP

Forty-two cells were followed from bud emergence to cytokinesis. Their vacuoles were visualized by phase-contrast microscopy and scored from time-lapse photomicrographs. The number of cells that showed the formation of the segregation structure from one point of the mother vacuole, and those that showed no visible vacuolar segregation structure, is indicated.

\begin{tabular}{lcc}
\hline \hline \multicolumn{1}{c}{ Stage } & $\begin{array}{c}\text { Time } \\
(\mathrm{min})\end{array}$ & $\begin{array}{c}\text { No. of } \\
\text { cells } \\
\text { scored }\end{array}$ \\
\hline $\begin{array}{l}\text { Point-like start of segregation structure seen } \\
\begin{array}{l}\text { Appearance of segregation structure after bud } \\
\text { emergence }\end{array}\end{array}$ & $5 \pm 6$ & 9 \\
$\begin{array}{l}\text { Disappearance of segregation structure after } \\
\text { bud emergence }\end{array}$ & $22 \pm 13$ & 34 \\
$\begin{array}{l}\text { No segregation structure seen } \\
\text { Generation time }\end{array}$ & $92 \pm 20^{*}$ & $20 \dagger$ \\
\hline \hline
\end{tabular}

* Scored from cytokinesis to cytokinesis.

$\dagger$ The remaining 22 cells could only be followed from bud emergence, not from the previous cell division.

existed for $10-45 \mathrm{~min}($ mean $=18 \mathrm{~min})$ in at least 34 cells. It was usually tubular but could also be an alignment of vesicles, particularly in cells with more fragmented vacuoles. Vesicle alignments remained for only a few minutes whereas tubular segregation structures usually lasted for a longer period. Vesicle alignments were sometimes an intermediate in the formation or breaking off of the tubular structures, but no fixed order could be established.

In eight cells the bud was seen to have a vacuole which was not preceded by a segregation structure. However, on photomicrographs these cells and their buds appeared 

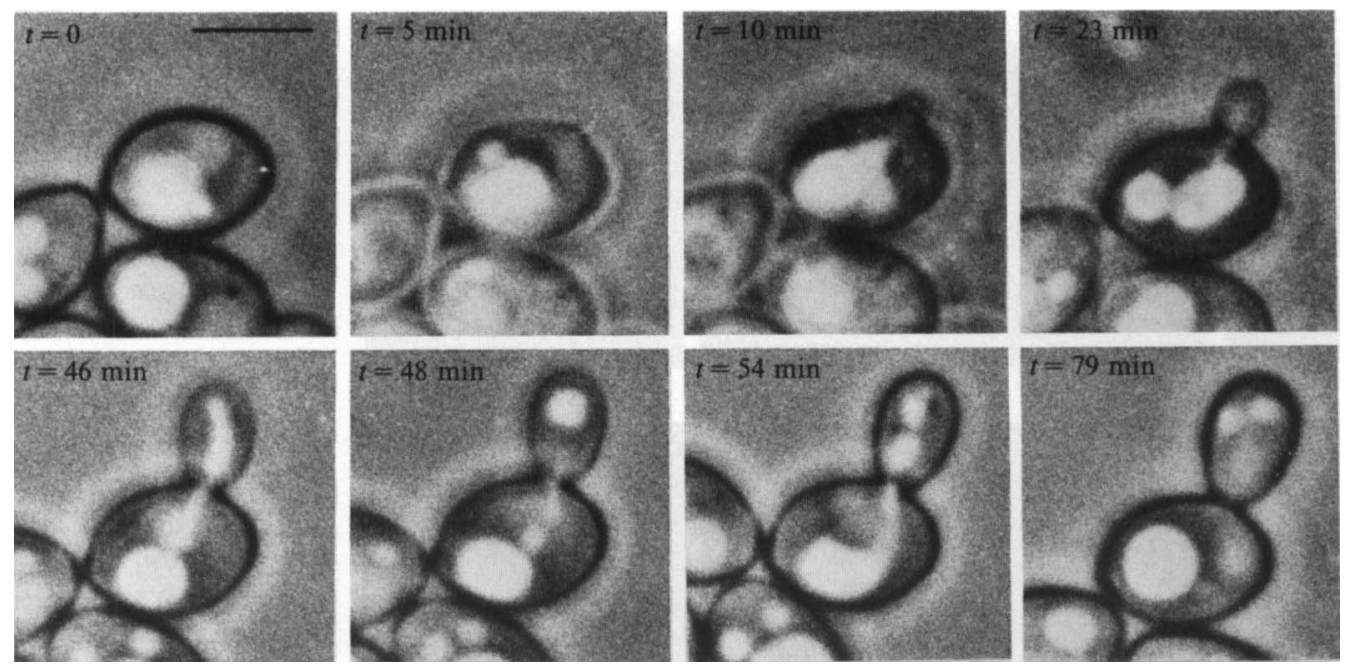

Fig. 2. Example of a time-lapse sequence of vacuolar segregation. Cells of X2180, growing on YPD-agar-PVP, were visualized by phase contrast microscopy. At $t=5 \mathrm{~min}$ bud emergence took place; at $t=10 \mathrm{~min}$ a segregation structure was formed from one point of the mother vacuole; at $t=48 \mathrm{~min}$ the segregation structure nearly disappeared, but at $t=54$ it had continued again; at $t=78 \mathrm{~min}$ the cell was in mitosis. Bar, $5 \mu \mathrm{m}$.

(a)

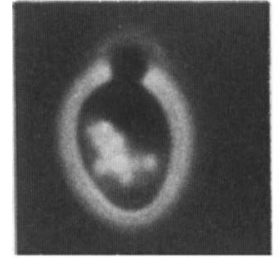

Category $1-$

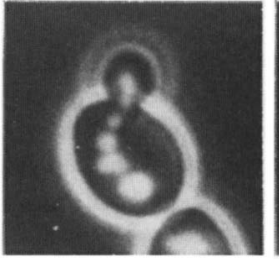

$2 a \curvearrowright$

(b)

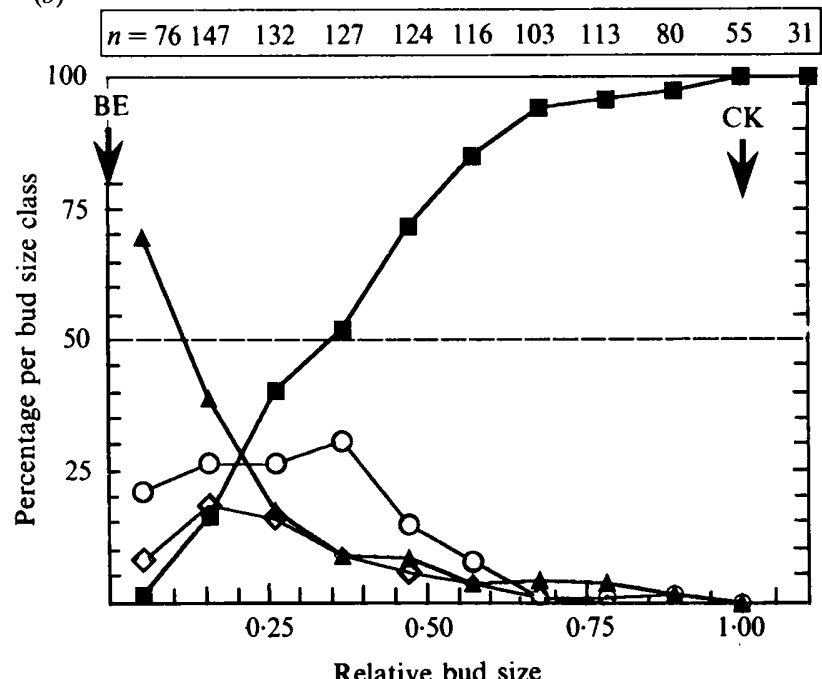

Fig. 3. Vacuolar morphologies of FITCstained X2180 cells were divided into three segregation categories and related to the bud size. Buds were classified by size and their value was normalized to one for the average bud size at cytokinesis. The number of cells per size class is indicated in the box; bud size classes containing less than 10 cells are not shown. Budded cells without a bud vacuole (category 1) were present especially in the cells with the smallest buds. Separate bud vacuoles (category 3) were present in the cells with the largest buds. Segregation structures, vesicular (category 2a) and tubular (category 2b), occurred in the cells with intermediate bud size, around 0.25 of the relative bud size at cytokinesis. The cells were scored from photomicrographs and bud sizes were determined as length $\times$ width. Abbreviations: BE, bud emergence; CK, bud size when $50 \%$ of the cells had passed cytokinesis. more out of focus than most other cells. Thus, they might have had a segregation structure that was out of focus, or persisting only for a short time, or of vesicles too small to be seen by phase-contrast microscopy. The above results are summarized in Table 1.
In some cells, a second segregation structure was formed after the first had disappeared; this could even occur a third time. In exceptional cases two segregation structures could occur at the same time (perceived in FITC-stained cells, see below). In nearly half of the cells 
(a)
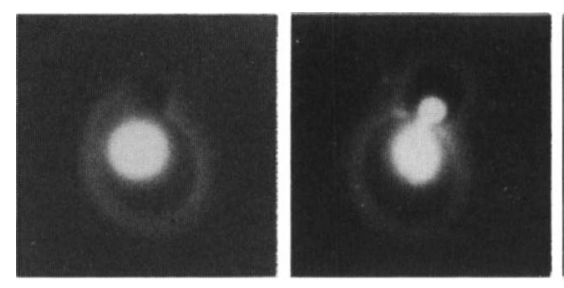

Category $1 \rightarrow$

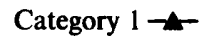

$2-$

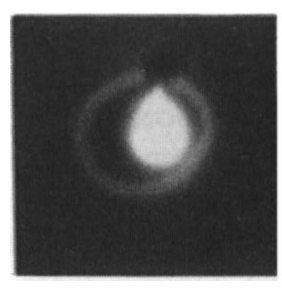

$1 \mathrm{a}-\Delta$

(b)

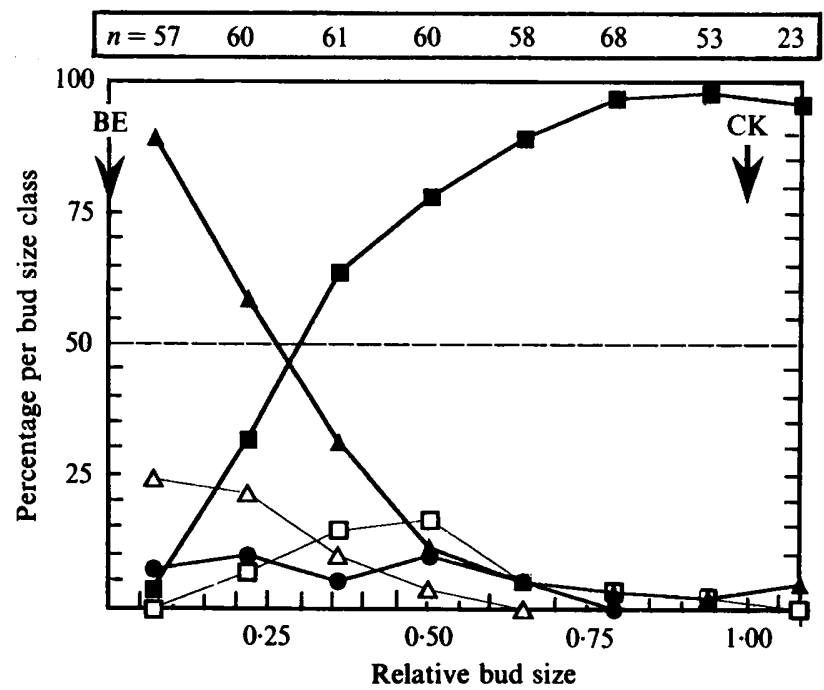

Fig. 4. Vacuolar morphologies of FITC-stained DL1 cells were divided into segregation categories and related to the bud size, as in Fig. 3. The number of cells per size class is indicated in the box; size classes containing less than 10 cells are not shown. Budded cells without a bud vacuole (category 1) comprised the majority of cells with small buds. Compared with X2180, large buds of DL1 still had no vacuole, since $50 \%$ of the buds have no vacuole at a relative bud size of 0.25 (instead of a relative bud size of $0 \cdot 12$ : see Fig. 3). Separate bud vacuoles (category 3) were present in cells with large buds, of the same relative size as in $\mathrm{X} 2180$. Cells were seen with a mother vacuole pointing towards the bud (category la) at bud sizes slightly smaller than at vacuolar segregation, and this was also seen in cells with a bud vacuole (category $3 a$ ), at bud sizes slightly larger than at vacuolar segregation. These cells are included in categories 1 and 3, respectively. The segregation structures were overrepresented in this figure since they were preferentially photographed. Abbreviations are defined in the legend to Fig. 3.

the nucleus could also be seen. The vacuolar segregation structure had disappeared in these cells before the nucleus migrated into the neck.
Strain differences in vacuolar segregation and morphology

As an alternative to the time-lapse study, the vacuolar segregation period was studied by classifying different stadia of vacuolar segregation as a function of bud size (Figs 3 and 4). The bud size was used as an indication of the relative position of the cell in the cell cycle. Exponentially growing X2180 cells were stained with FITC. As shown in Fig. 3, 70\% of the cells with the smallest buds had no vacuole in the bud (category 1 ). Half of the cells with somewhat bigger buds (with a bud size of about 0.25 relative to that at cytokinesis) had a vacuolar segregation structure either in the form of an alignment of vesicles (category 2a) or as a tubule (category 2b). A separate bud vacuole (category 3 ) was present in more than $90 \%$ of the cells with a relative bud size $>0.7$. These data show, like the time-lapse experiments, that cells acquire a vacuolar segregation structure prior to a separate bud vacuole. The tubular segregation structures and the vesicle alignments occurred in cells with the same bud size, but the tubular form was relatively more common, especially in cells with bigger buds. The reason might be that the tubule persisted longer than the vesicle alignment (see above), whereas they are formed simultaneously. That both structures are real and vesicle alignments are not partly unfocussed tubular vacuoles was confirmed by $3 \mathrm{D}$ confocal scanning laser microscopy, as illustrated in Fig. 5.

Vacuolar segregation in DLl was also studied in detail and exhibited a different morphology. DL1 usually had one large vacuole per cell. Its vacuolar segregation structure was always tubular and usually very short, with the mother vacuole lying near the neck (Fig. $4 a$, category 2). More than half of the cells with a relative bud size $<0.25$ had no vacuole in the bud (Fig. 4, category 1). Like $\mathrm{X} 2180$, in more than $90 \%$ of the cells with a relative bud size $>0.7$, a separate bud vacuole was present (category 3), so the segregation terminates at the same relative bud size in both strains. Cells without a bud vacuole were seen with the mother vacuoles pointing towards the bud (category 1a). They had slightly smaller buds than those at vacuolar segregation. Similarly, cells containing a separate bud vacuole and with the mother vacuoles pointing towards the bud (category 3a) had slightly larger buds than those at vacuolar segregation. Thus, the segregation structure appears to be formed from the mother vacuole and retracted into the mother vacuole when segregating stops.

An indication for the timing of vacuolar segregation in X2180 and DL1 can be obtained from Figs 3 and 4, respectively. The start is taken at the bud size where $50 \%$ of the cells have no vacuole in the bud and the end at the bud size where $50 \%$ have received a separate bud vacuole. From Figs 3 and 4 it is clear that DL1 has a very 

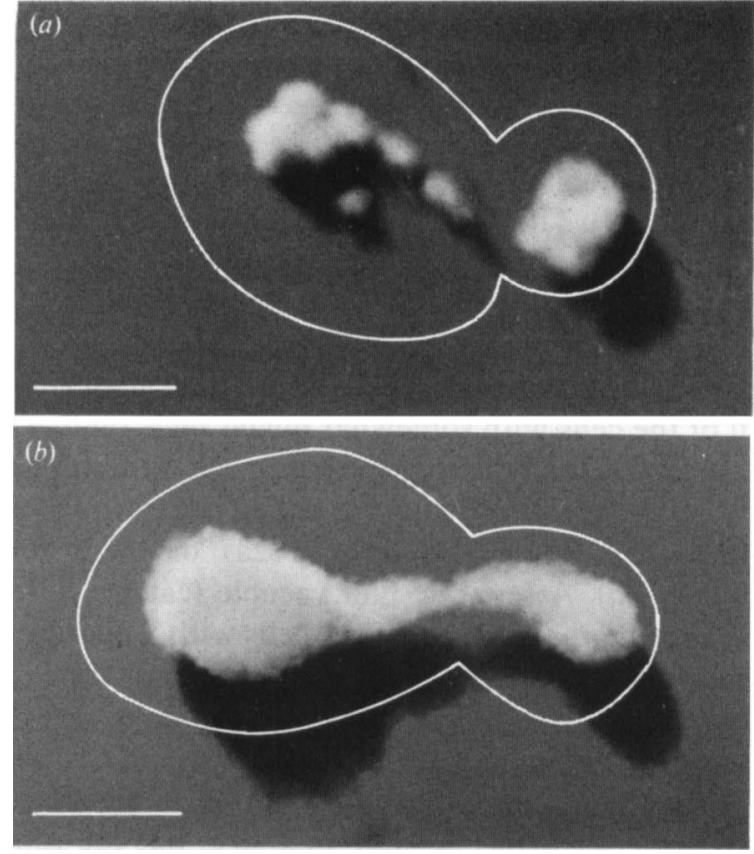

Fig. 5. Shadow images of two FITC-stained X2180 cells made with a confocal scanning laser microscope, showing $(a)$ a vesicle alignment and $(b)$ a tubular vacuole in the neck. Cells contours were obtained by negatively staining the cells with FITC in the surrounding medium. They are indicated separately since they are not visualized in shadow images, due to threshholding. Bars, $2.5 \mu \mathrm{m}$.

short-lasting segregation structure compared with $\mathrm{X} 2180$.

To confirm the general vacuolar shape and timing of segregation, three more strains were analysed: D27310B, DBY747 and S288C. Although none of the existing laboratory strains is known to be wild-type in every respect (Sherman, 1991), these strains were thought to be wild-types for vacuolar functions. All had fragmented vacuoles, resembling $\mathrm{X} 2180$, and their vacuolar segregation structures also resembled X2180. Therefore, DL1 probably has non-wild-type vacuoles. Remarkably, the vacuolar morphology of DL1 is similar to that of the mutants vps 3 and vacl, which segregate very little vacuolar material to the bud (Raymond et al., 1990). DL1 cells also segregated smaller vacuoles into the buds than the other four strains.

By analogy with nuclear migration, the equations from Tyson et al. (1979) were used to calculate the timing of vacuolar segregation. The average start of vacuolar segregation was calculated from the percentage of budded cells without a bud vacuole in exponentially growing cultures. In the different strains the average start of vacuolar segregation varied between 3 and $17 \mathrm{~min}$ after bud emergence. The duration of vacuolar segregation was calculated from the portion of cells with a vacuolar segregation structure and varied between
4 min (in DL1) and 32 min (in DBY747; see Fig. 6). Although the duration varied greatly, the vacuolar segregation took place at approximately one-fifth of the way into budded phase in all strains.

\section{Nocodazole does not affect vacuolar segregation}

The effect of nocodazole on vacuolar morphology was studied in X2180 and DL1. The vacuoles of X2180 were fragmented by nocodazole within $30 \mathrm{~min}$ as shown by Guthrie \& Wickner (1988). Surprisingly, nocodazole did not fragment the vacuoles in DL1 (results not shown).

To show the effect of nocodazole on vacuolar segregation, both strains were synchronized by elutriation. Fractions enriched with unbudded cells $(>50 \%)$, were incubated with nocodazole and FITC, and followed during one cell cycle. Two controls were set up: one without nocodazole and FITC, and one with nocodazole without FITC. Samples were taken at different times and fixed with formaldehyde to measure cell numbers and nuclear division by DAPI staining. Nocodazole prevented cell division and nuclear division, but not bud emergence, corresponding with the results of Jacobs $e t$ al. (1988). Shortly after bud emergence in X2180 and after about $20 \mathrm{~min}$ in DL1, many vacuolar segregation structures were seen. In both strains more than $90 \%$ of the cells stopped further cell cycles as large budded cells with a single nucleus near the neck, and with separate vacuoles in mother cell and bud. Since nocodazole stopped nuclear division it presumably depolymerized the microtubules. Thus, vacuolar segregation is probably independent of microtubules.

\section{Discussion}

From the time-lapse experiment, summarized in Table 1, it has been shown, using phase-contrast microscopy, that the vacuolar segregation structure proceeds from one point of the mother vacuole. Like the nucleus, vacuoles are divided in a discrete cell cycle step, prior to nuclear migration. However, the process does not have the precision of nuclear division: more than one vacuolar segregation structure can occur in one cell cycle. Furthermore, vacuolar segregation can take place either by a tubular vacuolar extension or by an alignment of vacuolar vesicles through the neck. The vacuolar segregation structure has already been partly characterized by Raymond et al. (1990) and it is similar to the intervacuole vesicular traffic seen in zygotes; this can also consist of either a tubule or vesicles (Weisman \& Wickner, 1988; Weisman et al., 1990).

Vacuolar segregation in X2180 started $5 \mathrm{~min}$ after bud emergence. It lasted on average for 14 or $17 \mathrm{~min}$, as 


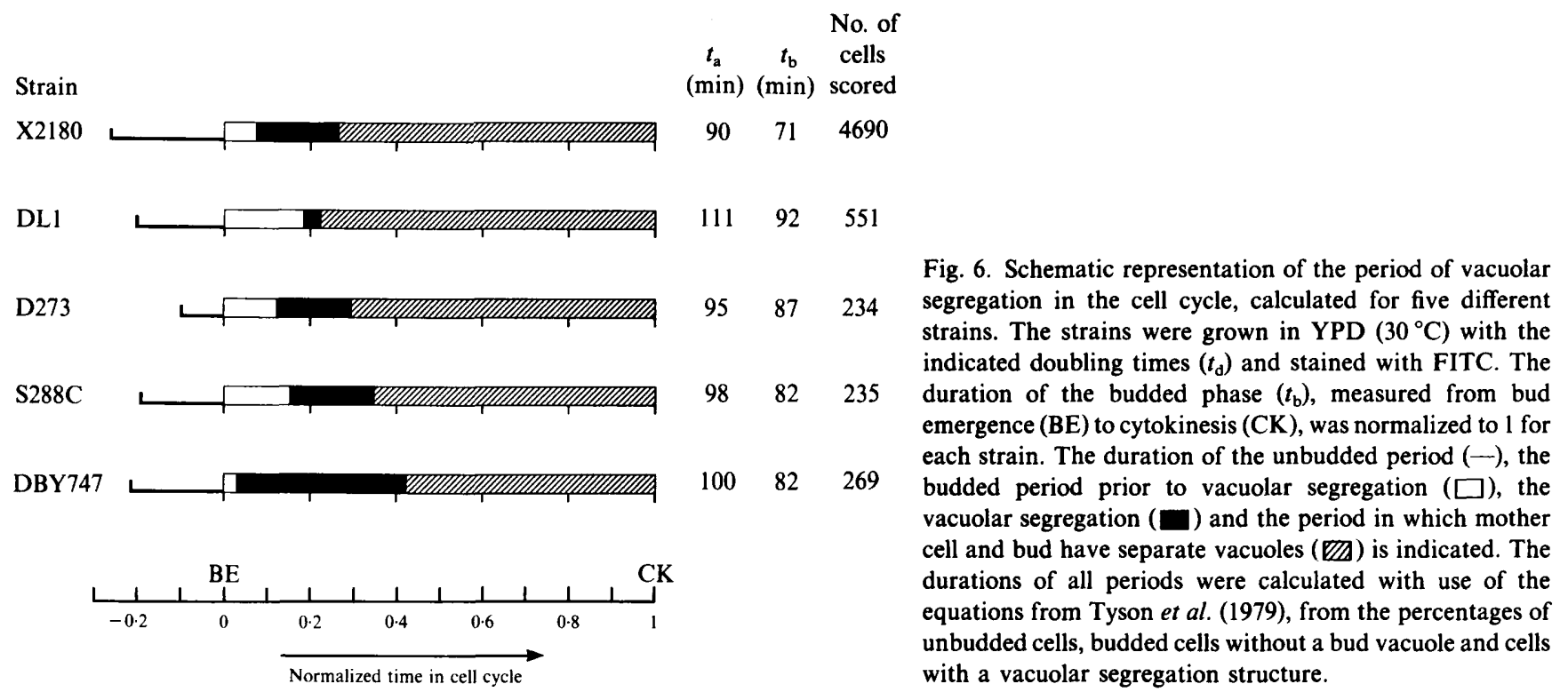

measured from the percentage of segregation structures in exponentially growing culture or from the time-lapse experiment, respectively. Different strains varied in timing and duration of vacuolar segregation in the cell cycle (Fig. 6). However, all the strains we tested have segregation structures which occur in the first half of the budded period. Segregation structures were even seen in a small percentage of the vacl-1 mutant (LWY148; data not shown), which segregates only a very small vacuole into the bud (Weisman et al., 1990). Vacuoles also segregated into the multiple buds of a $c d c 4$ mutant at the restrictive temperature (data not shown), so the process seems to be independent of DNA replication.

Vacuolar morphology differed in various strains, but most commonly it consisted of $2-30$ vesicles in a cluster. Although DLl was thought to be a wild-type strain except for some auxotrophies, it had a slower growth rate and a more coherent vacuole than the other strains. Furthermore, it had a brief vacuolar segregation period (Fig. 6) and often a relatively small vacuole in the bud. Diploids from DL1 and X2180-1A have vacuoles like X2180 (results not shown). Therefore, DL1 might have a recessive mutation similar to the vps 3 and vacl mutants, but less extreme. The vps 3 and vacl mutants are thought to sometimes perform cell division before a vacuole is seen in the bud. In DLl all buds received a vacuole before cell division, albeit sometimes a small one; perhaps vps 3 and vacl also have vacuoles in all buds, but these are sometimes too small to be seen by light microscopy.

Nocodazole was previously shown to cause fragmentation of vacuoles in X2180 and a nocodazole-resistant

tub2-201 mutant also had fragmented vacuoles (Guthrie \& Wickner, 1988). This could be repeated in the X2180 strain, but did not occur in DL1, so it might be an indirect effect. In both strains nocodazole blocked nuclear division, but did not affect vacuolar segregation. This makes microtubule-directed vacuolar segregation unlikely and affirms recent ideas that yeast microtubules are exclusively involved in nuclear processes (Stearns, 1990). Since the segregation structure is formed from the mother vacuole from one point and directed to the bud, motor proteins attached to other cytoskeleton elements, such as myosin and actin, might be involved.

We gratefully acknowledge Peter Huls for synchronizing cells, Jos Grimbergen for the CSLM images, Chris Molenaar and Karin Visser for experimental assistance, and Norbert Vischer, Koen Visscher and Michel van Bakel for technical assistance. We are grateful to Lois Weisman for generously providing the vacl-1 mutant.

\section{References}

Banta, L. M., Vida, T. H., Herman, P. K. \& EmR, S. D. (1990). Characterization of yeast $\mathrm{Vps} 33 \mathrm{p}$, a protein required for vacuolar protein sorting and vacuolar biogenesis. Molecular and Cellular Biology 10, 4638-4649.

Brakenhoff, G. J., van Spronsen, E. A., van der Voort, H. T. M. \& NanNinga, N. (1989). Three-dimensional confocal fluorescence microscopy. Methods in Cell Biology 30B, 379-398.

Gonzalez, R. C. \& WinTZ, P. (1987). Digital Image Processing. Reading, MA: Addison-Wesley.

GUTHRIE, B. A. \& WICKNER, W. (1988). Yeast vacuoles fragment when microtubuli are disrupted. Journal of Cell Biology 107, 115-120.

Herman, P. K. \& EMR, S. D. (1990). Characterization of VPS34, a gene required for vacuolar protein sorting and vacuole segregation in Saccharomyces cerevisiae. Molecular and Cellular Biology 10, 67426754. 
Jacobs, C. W., Adams, A. E. M., Staniszlo, P. J. \& Pringle, J. R. (1988). Functions of microtubules in the Saccharomyces cerevisiae cell cycle. Journal of Cell Biology 107, 1409-1426.

Klionsky, D. J., Herman, P. K. \& EmR, S. D. (1990). The fungal vacuole: composition, function and biogenesis. Microbiological Reviews 54, 266-292.

LORD, P. G. \& WhEALS, A. E. (1981). Variability in individual cell cycles of Saccharomyces cerevisiae. Journal of Cell Science 50, 361376.

Preston, R. A., Murphy, R. \& Jones, E. W. (1987). Apparent endocytosis of fluorescein isothiocyanate-conjugated dextran by Saccharomyces cerevisiae reflects uptake of low molecular weight impurities, not dextran. Journal of Cell Biology 105, 1981-1987.

Pringle, J. R., Preston, R. A., Adams, A. E. M., Stearns, T. Drubin, D. G., HaARer, B. K. \& Jones, E. W. (1989). Fluorescence microscopy methods for yeast. Methods in Cell Biology 31, 357-435.

RAYMOND, C. K., O'Hara, P. J., Eichinger, G., Rothman, J. H. \& STEVENS, T. H. (1990). Molecular analysis of the yeast VPS3 gene and the role of its product in vacuolar protein sorting and vacuolar segregation during the cell cycle. Journal of Cell Biology 111, 877-892.

RoBinow, C. F. (1975). The preparation of yeasts for light microscopy. Methods in Cell Biology, 11, 1-22.

SHERMAN, F. (1991). Getting started with yeast. Methods in Enzymology 194, 3-21.
StEARNS, T. (1990). The yeast microtubule cytoskeleton: genetic approaches to structure and function. Cell Motility and the Cytoskeleton 15, 1-6.

TYSON, B. T., LORD, P. G. \& WhEALS, A. E. (1979). Dependency of size of Saccharomyces cerevisiae cells on growth rate. Journal of Bacteriology 138, 92-98.

Van Loon, A. P. G. M., van Eijk, E. \& Grivell, L. A. (1983). Biosynthesis of the ubiquinol-cytochrome $c$ reductase complex in yeast. Discoordinate synthesis of the $11-\mathrm{kD}$ subunit in response to increased gene copy number. EMBO Journal 2, 1765-1770.

VAN DER Voort, H. T. M., Brakenhoff, G. J. \& BaARslaG, M. W. (1988). Three-dimensional visualization methods for confocal microscopy. Journal of Microscopy 153, 123-132.

WEISMAN, L. S. \& WICKNER, W. (1988). Intervacuole exchange in the yeast zygote: a new pathway in organelle communication. Science 241, 589-591.

Weisman, L. S., Bacallao, R. \& Wickner, W. (1987). Multiple methods of visualizing the yest vacuole permits evaluation of its morphology and inheritance during the cell cycle. Journal of Cell Biology 105, 1539-1547.

Weisman, L. S., EmR, S. D. \& Wickner, W. (1990). Mutants of Saccharomyces cerevisiae that block intervacuole vesicular traffic and vacuole division and segregation. Proceedings of the National Academy of Sciences of the United States of America 87, 1076-1080. 